\title{
Process for enhancing the production system robustness with sensor data - a food manufacturer case study
}

\author{
Sofie Bech ${ }^{1}$, Thomas Ditlev Brunoe ${ }^{1}$ and Kjeld Nielsen ${ }^{1}$ \\ ${ }^{1}$ Department of Materials and Production, \\ Aalborg University, Fibigerstrade 16, DK9220 Aalborg, Denmark \\ sofie@mp.aau.dk
}

\begin{abstract}
Global markets for food products are changing towards accommodating larger product variety while experiencing shorter lifespans of products. These market trends impose pressure on the existing manufacturing infrastructure, however the food industry lacks investment in manufacturing technology. This research illustrates how the robustness of the existing production system can be enhanced using sensor data, including a case study of a Danish food company. The paper presents an outline of an iterative process performed in a case company to identify and carry out the potential of utilize sensor data from the existing production setup, and an example of the potential and return of investment is introduced. Several challenges are recognized when implementing sensor data to enhance the production system robustness. The in-house competencies are lacking in order to install the setup of sensors and operating in a database with a big amount of data. In addition, the challenge of continuously maintaining the data and the analyses is present. The research points to the importance of involving operators to better understand the context of the production. In conclusion, the case company has achieved information of the production based on data and not only running adjustments, based intuition of the operators and has thereby enhanced the production system robustness.
\end{abstract}

Keywords: Food manufacturing, Sensor data, Production system robustness

\section{Introduction}

Within the food industry, global markets are today driven by increasing global competition, and a demand for changes in product mix, and frequent introductions of new products $[1,2]$. In addition, the food and beverage markets has shifted towards a very dynamic market, with increasing competition, lower profit margins and extensive new regulations $[3,4]$. Nevertheless, in many companies, the food and beverage production setup has difficulties accommodating these new challenges partly due to the production setup being designed and operated with tacit knowledge and the production equipment has a long service life [5]. Another aspect is lower investments in manufacturing technology compared to other industries. As an indicator of this lacking investment, in 2016 the food industry, commissioned 8,200 industrial robots worldwide, while the 
automotive industry commissioned 103,300 units in 2016 accounting for a share of $35 \%$ of the total supply of industrial robots [6]. Moreover, Kohr et al. [7] state that in comparison, the automotive OEM industry has a relation of $30 \%$ of sites of R\&D vs production sites, whereas the process industry has only a $11 \%$ ratio.

This difference in investments and R\&D indicates that food and beverage companies are less likely to design and commission new manufacturing lines, when introducing new products and variants. Hence, new products need to be introduced in existing manufacturing systems, which yields a requirement for higher process robustness. The majority of research on designing robust processes focuses on establishing new manufacturing systems, i.e. green field operations. Green field research in production robustness thus cannot be applied directly due to the lack of willingness to invest in new equipment. Therefore, to accommodate the new market demand with existing equipment, this research addresses whether sensors can be utilized to collect data from the existing manufacturing equipment to increase process robustness. As the cost of sensors setup in manufacturing has decreased over the last few years, this has given way for this opportunity. The challenges and opportunities outlined in this section leads to the research question of this paper:

How can robustness of the existing production systems be enhanced, using sensor data?

\section{$2 \quad$ Method}

To address the research question, a case study is conducted within a Danish food manufacturing company. The methodology of case study is selected due to needed for analyzing the phenomenon of sensor data of the existing production system, in its specific context (the food manufacturing industry) $[8,9]$. The case company is introduced in section 2.1. The research takes an outset in a two-year project in the case company where the authors participated. A new process for establishing sensor data acquisition, forming and testing hypotheses and transforming those into actions to improve the manufacturing system is proposed by the authors and outlined in section 3 . This process is combined with the case study to exemplify the process and determine the potential in the specific case. The contribution of the research is thus twofold: 1) a proposed iterative method for establishing sensor-based data collection in food manufacturing, and 2) a case study outlining the application of the method and examples of actual improvements in the manufacturing system suggested by the method.

\subsection{Case company}

The case study was conducted in a Danish manufacturer of Danish pastry. The company has two separate production lines with a total annual production of 12.700 tons. The products have different recipes, fillings, toppings, and shapes, implying thousands of possible combinations. Nevertheless, a the process flow is on an overall level generic, and is illustrated in Fig. 1, including the following activities: 1) mixing of the dough, 2) processing of dough and incorporating margarine, 3) shaping of products and adding filling and topping, 4) raising in a warm environment and 5) freezing. 


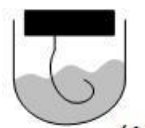

(1)

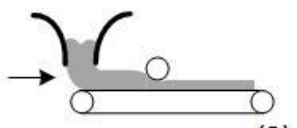

(2)

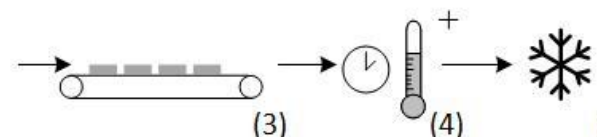

(3)
(5)

Fig. 1. A generic process flow at the case company [10]

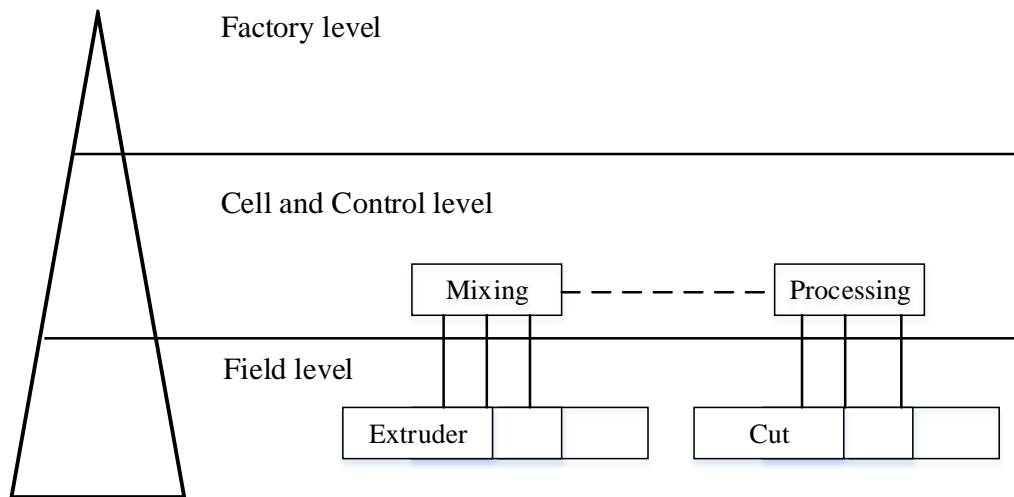

Fig. 2. The information flow at the case company

Fig. 2. Illustrates a section of the previous information flow at the case company, structured according to the automation pyramid $[11,12]$. The boxes represent the systems that operate on different levels, and lines represent flow of information. There is a dedicated system controlling the mixing process of the dough which has a direct connection with the PLC on the field level, which is the general picture of most processes in the production lines. The factory level addresses the overall business processes and the supply management, and at this level the data is only required for production planning etc. Today, at the case company the information flow between the factory level and the cell and control level sparse and rarely utilized. Specifically, performance data which could be utilized for optimizing the production planning and control is not implemented. The communication between the factory level and cell and control level is primarily manual, and no direct integration is implemented. Hence, information primarily flows from factory level and down, whereas no information formally flows from the lower levels to factory level. This implies that decisions on factory level are not able to be supported by information from the lower levels. On cell level and control level, part of the lines are controlled by individual systems and limited to no communication is done between these systems. This sparse communication is illustrated by a dotted line in Fig. 1. Moreover, the systems on cell level and control level are mainly manually controlled by an operator. Since little information on machine settings is formalized, 
this results in a production system run on tacit knowledge of how the operator intuitively thinks the process should be controlled, and a system with little fact based justification of decisions on higher levels.

\section{Identifying the Potential}

As stated in the introduction, the food industry generally lacks behind other industries in terms investing in utilizing sensor data for improving operations. Although adding sensors to existing production systems is an inexpensive alternative to establishing new more digitized production systems, identifying how the sensor data can improve the processes is a major task in the case company. Moreover, determining the potential outcome on beforehand is a time-consuming task, and thus the proposed process is iterative, encouraging experimentation with data from inexpensive sensors. Figure 3 illustrates the process from initial hypotheses to conclusion and capitalization. In the following, each part of the process in fig. 3 is outlined. 1) The process starts with hypotheses, stated by experts in the company, of what the potentials might be of using sensor data in the existing production system, and what data is necessary to address these hypotheses. Establishing the hypotheses involves several parts of the company, including operators, technicians, engineers and data analysts. The initial hypotheses thus imply requirements for new sensors. 2) The data needed, and quality requirements for the data depends on the hypotheses. There are basically two solutions to obtain data of the existing production equipment: a) Adding new sensors or b) accessing existing sensors in existing equipment. When adding new sensors to existing equipment, the location of the sensors and type of sensors must be carefully considered. With a tough, and strongly regulated production environment as the food industry, all sensors must stand a cleaning. Next, the sampling frequency of data logging must be determined, so that the information is sufficient but not excessive. 3) Collect sensor data. Once sensors are installed, and integrated into computer network, all sensor data is stored in one common database, which is made available to different stakeholders within the company. The timespan needed is implied by the initial hypotheses. 4/5) Analyzing the data to address the hypotheses. If data is logged by using existing equipment PLCs, there is a task of translating the names in the PLC into terms that are familiar to stakeholders, since there may be a lack of documentation of the existing equipment. If the production line consists of both a continuous and a discrete flow, there is a task of relating the data to the same timeframe, especially when the production process is operator driven. Once this is completed the actual analyses can be performed which should be done by applying data analytics software, such as R Studio, Microsoft Power BI or a combination, depending on the actual analyses necessary to address the hypotheses. If the hypotheses can be addressed, the analyses can be concluded and results can be capitalized on, and the loop may be terminated. Alternatively, if the hypotheses are rejected, new hypotheses can be formulated and evaluated through a new loop.

6) As a side effect of this process, questions can be raised of new potentials. Moreover, as a spinoff from the analyses new hypotheses may be generated. If the data foundation is sufficient for the new analyses, this can lead to new findings through data 
analytics. If not, the process takes a new loop as illustrated in Fig 3. by establishing sensor data collection, collect sensor data, and analyzing data. Chapter 4 presents an example application from the case company.

\section{Example}

This section presents an example from the case company applying the method illustrated in fig 3. From the initial hypotheses to establish sensor data, collect sensor data for the analyses and conclude and capitalize. Furthermore, this example includes figures on cost and savings as a result of applying the method.

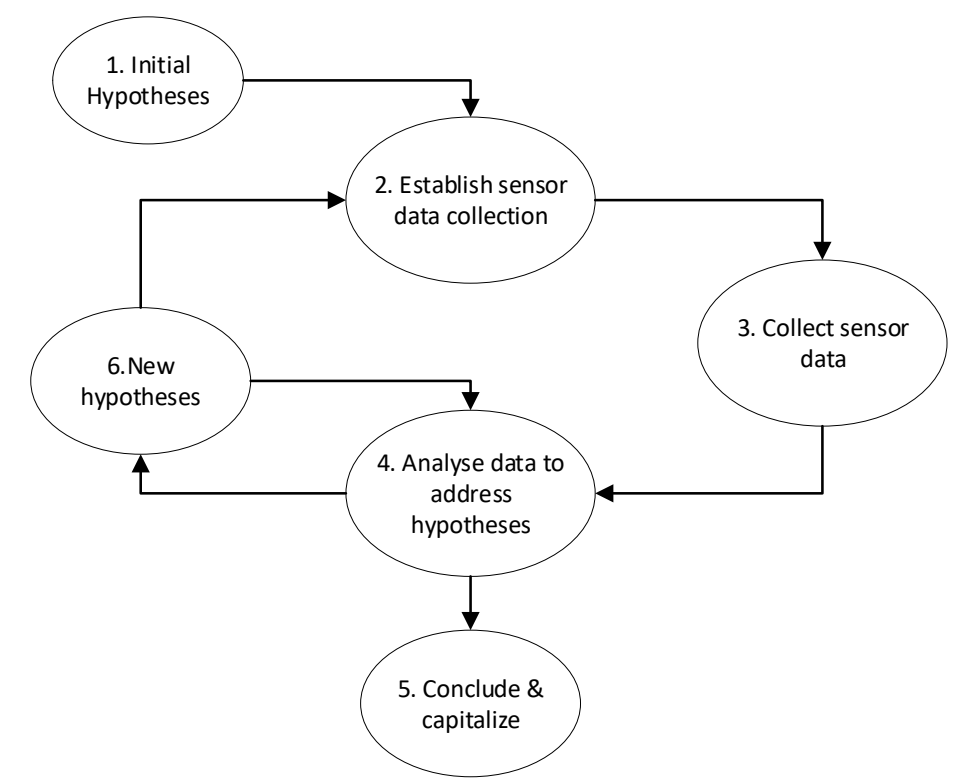

Fig. 3. The process of establishing sensor data collection, analyzing and capitalizing on using sensor data

\subsection{Identifying start temperature}

Temperature is key to producing high quality Danish pastry. Prior to this project, only the initial temperature of the dough was measured by a manual offline test. One operator was in control of mixing the dough and had a recipe defining guidelines for the temperature of the dough. This can vary from 4 degrees $\mathrm{C}$ to 7 degrees $\mathrm{C}$ regardless of the ambient temperature in the factory. The operator can add $\mathrm{CO}_{2}$ manually and thereby control the initial temperature of the dough. The optimum start temperature of the dough has been a topic of discussion for several years. The hypotheses formulated by the stakeholders on the case company was: Controlling the initial temperature of the dough based on sensor data can reduce the $\mathrm{CO} 2$ consumption. The process of establishing sensor data started by looking into the current production setup. Temperature 
sensors were added to the system with the purpose of understanding the effect on the temperature. Three online sensors measuring the dough temperature were added, and two sensors were implemented in order to log the ambient temperature in the factory. The data collection had a 10 month time-span with a frequency of one set of data points per minute. The frequency of the data logging was determined by interviewing operators determining how often changes were made. The logged data was analyzed by loading the dataset into Microsoft Power BI, applying the R plugin for advanced statistical analyzes. Following this, a correlation analysis was made determining the correlation between the initial temperature of the dough, dough temperatures further along the process chain, the ambient temperature and the amount of cooling $\mathrm{CO} 2$ injected into the dough. The analysis indicated that the initial temperature of the dough in many cases had been lower than actually necessary, and determined a clear correlation between the ambient temperature and the increase in dough temperature. This correlation was translated into a table indicating how much $\mathrm{CO} 2$ should be injected at different combinations of ambient temperatures and initial dough temperatures in order to achieve the desired dough temperature along the production line. In conclusion, the hypothesis was confirmed, since using temperature sensor data from the production it was now possible to define the optimal start temperature of the dough and determine the right amount of $\mathrm{CO} 2$.

\subsection{Cost of investment}

The initial investment included new sensors, installation, documentation and connection to a database. The direct cost of that was 30.000 DKK (4,500 USD). The return of investment is directly related to a reduction of $\mathrm{CO}_{2}$ injected. Today the annual cost $\mathrm{CO}_{2}$ used for regulating dough temperature is approximately 1 million DKK. However, by introducing acceptance of a higher initial temperature of the dough, $\mathrm{CO}_{2}$ consumption can be reduced by 30-40\%. Therefore, solely based on the $\mathrm{CO}_{2}$ reduction, this investment has an approximated return on investment of roughly one month. As an additional benefit of higher initial dough temperature, other analysesa indicate a quality increase of the final product. Moreover, these analyses also indicated fewer production stops and less product waste due to the increase in quality.

As a side effect of this analysis, new potentials were identified. rather than controlling the initial dough temperature in the processing part of the production, it raises the question of controlling the temperature earlier in the process. In other words, going from reacting based on the temperature measured to predicting the amount of $\mathrm{CO}_{2}$. This prediction can be based on the ambient temperature and the temperature of the ingredients. Thereby, controlling the temperature based on the real-time data with a direct feedback loop. However, to make this analysis new sensors have to be added to the flour silo and the water dispenser. This is currently an ongoing project at the case company. 


\section{Conclusion}

The increasingly dynamic markets impose pressure on food and beverage manufacturers but investment in new state of the art production lines are in some cases not possible, and the industry generally lacks investments in new technology. The aim of this research was to identify how sensor data from the existing production system can be utilized to achieve a more robust production system, and to address this, an iterative process for establishing sensor data collection and analyses was introduced, and a case study has been conducted within a Danish food manufacturing company. In addition, an example of applying the method in the case company was included. By introducing sensors in the production in the case company, the knowledge of how temperature affects the dough and the case company can reduce the amount of $\mathrm{CO}_{2}$ used for cooling was established. This part of the project had a return of investment of one month and as a potential side effect a quality increase in the product. Moreover, further recommendations for applying sensors and data analyses, found from this research are outlined below:

Extend in house competences: Installing the setup of sensors, establishing a link to in a database with a big amount of data, and navigating this data requires special competences. This research revealed a lack in the in-house competences in the case company. Thus, the case company has outsourced all setup of data logging and database development. However, still it is a major learning curve in making value adding analyses with more than 10.000 .000 data points.

Maintain the analyses: Using data as the foundation of decisions in the production, calls for continuous maintenance of the analyses / assumptions and formulation of new hypotheses

The importance of including operators: in order to involve the operators in linking the data into a complex production context in the case company, several interviews have been performed, thereby relating the data to specific challenges in the production. This proved to be a significant change management task.

Possibility for having a fact based discussion of the production, across management: Having production data changes the focus from operator intuition driven production to rather data driven production. Reducing the operator dependency introduces better quality of the products due to more stable process.

Applying the method proposed in this paper in the case company resulted in more robust manufacturing processes. Being in control of manufacturing processes and increasing robustness is expected to allow the company to increase product variety and reduce time to market, providing a more agile manufacturing setup fit for serving markets requiring increased variety and more frequent product introductions.

\section{References}

1. Koren, Y., Shpitalni, M.: Design of reconfigurable manufacturing systems. J.Manuf.Syst. 29(4), 141 (2010). 
2. Wiendahl, H., ElMaraghy, H. A., Nyhuis, P., Zäh, M. F., Wiendahl, H., Duffie, N.,

Brieke, M.: Changeable manufacturing-classification, design and operation. 56(2), 783-809 (2007).

3. OpenText: Enterprise information management for the food and beverage industry. (2013).

4. Gargouri, E., Hammadi, S., Borne, P.: A study of scheduling problem in agro-food manufacturing systems. Math.Comput.Simul. 60(3-5), 291 (2002).

5. McIntosh, R. I., Matthews, J., Mullineux, G., Medland, A. J.: Late customisation: Issues of mass customisation in the food industry. 48(6), 1557-1574 (2010).

6. International Federation of Robotics: Executive summary world robotics 2017 industrial robots\&nbsp;. https://ifr.org/downloads/press/Executive_Sum-

mary_WR_2017_Industrial_Robots.pdf.

7. Kohr, D., Budde, L., Friedli, T.: Identifying complexity drivers in discrete manufacturing and process industry. 63, 52-57 (2017).

8. Voss, C.: Case research in operations management. In: Researching operations management, pp. 176-209. Routledge (2010).

9. Eisenhardt, K. M., Graebner, M. E.: Theory building from cases: Opportunities and challenges. 50(1), 25-32 (2007).

10. Bech, S., Brunoe, T. D., Larsen, J. K.: Changeability of the manufacturing systems in the food industry - A case study. (2018).

11. Schilberg, D., Meisen, T., Reinhard, R.: Virtual production Intelligence-Process analysis in the production planning phase. In: Engineering education 4.0, pp. 131-144. Springer (2016).

12. Trsek, H.: Isochronous wireless network for real-time communication in industrial automation. Springer (2016). 